\title{
Log Periodic Dipole Antenna Design Using Particle Swarm Optimization
}

\author{
M. Aziz-ul-Haq ${ }^{1, *}$, M. Tausif Afzal ${ }^{1}$, Umair Rafique ${ }^{1}$, Qamar-ud-Din $^{2}$, M. Arif Khan ${ }^{1}$, M Mansoor Ahmed $^{2}$ \\ ${ }^{1}$ Department of Electronic Engineering, Mohammad Ali Jinnah University, Islamabad, 44000, Pakistan \\ ${ }^{2}$ Department of Electrical Engineering, University of Faisalabad, Faisalabad, 38000, Pakistan
}

\begin{abstract}
This paper presents an optimized design of Log Periodic Dipole Antenna (LPDA) for GSM phase (I and II), WiMAX, Bluetooth $^{\circledR}$, Wi-Fi and $3 \mathrm{G}$ mobile communication bands. First, the LPDA is designed by evaluating its parameters and then it is optimized by using evolutionary technique called Particle Swarm Optimization (PSO). It is observed that in optimized design, the VSWR is closed to its ideal value i.e. 1. Moreover, the gain is improved up to an average of $0.6 \mathrm{~dB}$ as compared to the initial gain. It is also noticed that the optimized design is physically of smaller size as compared to the initial one. It gives an ease in implementation which is consequently cost effective.
\end{abstract}

Keywords Log Periodic Dipole Antenna, Particle Swarm Optimization, Design Reduction, Cost Effective

\section{Introduction}

The transmission of data at high rate is the basic need in modern communication system. For this purpose, the frequency independent antenna with coverage of wide range of frequencies is the ultimate goal. One of the most powerful and popular method for such type of applications is the designing of Log Periodic Dipole Antenna (LPDA) which was the endeavour of Isbell and Carrel[1,2].

A Log Periodic Dipole Antenna is a wideband antenna that is not affected by changing the spectrum of frequencies within the desired band[3]. LPDA consist of number of elements having unequal lengths, spacing and diameters. The prelursory work of $[1,2]$ presented the precise designing of LPDA. The basic response of LPDA has been analysed by the mathematical formulas introduced by[1,2]. The behaviour of current across each dipole element is the main task for the response of LPDA. The prerequisite condition described by[4] which was used to find current and impedance between parallel antennas of unequal lengths.

In the mid 1990's, the evolutionary technique was introduced by Kennedy and Eberhart[5] namely Particle Swarm Optimization (PSO). The use of this algorithm is a very smart approach to many electromagnetic problems. This algorithm has been used due to fast convergence, simple implementation, less computation and generally few lines of code[6-7]. There are numerous optimization techniques are frequently applied in many electromagnetic related problems[8-15]. Out of those techniques, PSO and its different

* Corresponding author:

muhammad.azizulhaq@yahoo.com (M. Aziz-ul-Haq)

Published online at http://journal.sapub.org/ijea

Copyright (C) 2012 Scientific \& Academic Publishing. All Rights Reserved varia- nts are considered powerful optimization tools which offer almost guaranteed convergence for complex problems[5-6].

With the aid of $[1,2]$, we calculate the basic response of LPDA and then this design is optimized using PSO. This technique minimizes the design of LPDA as compared to the initial design. Moreover, it is observed that how this computational algorithm alters the gain and VSWR of Log Periodic Dipole Antenna as compared to the standard of LPDA.

\section{Design and Simulation of LPDA}

Log Periodic Dipole Antenna (LPDA) has some endless benefits which are high gain and optimum VSWR. Due to these benefits, it is extensively used in many applications. LPDA was first introduced in University of Illinios by Carrel in 1960[2].

The schematic diagram of LPDA is shown in Fig. 1 which consists of number of dipole elements having unequal lengths, spacing and diameters. The step by step design procedure of LPDA is described by[16-17]. The basic design equations are used to determine different parameters of LPDA which are given below

$$
\begin{gathered}
\sigma=\frac{d_{n}}{2 L_{n}} \\
L_{n+1}=L_{n} \times \tau \\
\alpha=a \tan \left(\frac{1-\tau}{4 \sigma}\right) \\
X_{n}=h_{n} \times \tan (\alpha)
\end{gathered}
$$

The spacing between every two consecutive elements is determined by using eq. (2). $\sigma$ is a spacing factor in the range of $0.04 \leq \sigma \leq 0.22[18]$. Eq. (3) is used to determine the length of each element in which $\tau$ is a scaling factor in the range of 
$0.76 \leq \tau \leq 1$. The distance from source to any dipole element is determined by eq. (4). The initial response of LPDA is observed against the design using $\tau=0.9, \sigma=0.16, N=13$, $L_{n} / d_{n}=125, Z_{o}=100 \Omega$ and $L_{l}=\lambda_{\text {ref }} \times 0.5$, respectively. Where, $\lambda_{\text {ref }}$ is calculated from reference frequency which is $400 \mathrm{MHz}$ in our case. This reference frequency is used to define the length of first dipole element. The rest of the lengths of each dipole element and other parameters are calculated from the design equations which discussed above. The design parameters corresponding to each dipole element are given in Table 1.

Where, $h_{n}$ is the length of $n^{\text {th }}$ element and $X_{n}$ is the distance from source to $n^{\text {th }}$ dipole element, respectively. The response is being calculated as discussed in[17]. The important factor is to determine the current across each dipole element which can be determined by knowing the impedance of each dipole element and admittance of feeder line as step by step procedure which discussed in[17]. The method of moment is being used to determine the impedance across each dipole element[4]. The admittance of feeder line is determined by a method as discussed in[2]. The initial response is taken by implementing a MATLAB routine against the design parameters. The initial response is compared with[18] which include gain and VSWR. The initial gain for different communication bands are shown in Fig. 2-5. The overall initial VSWR for the reported bands is shown in Fig. 6.

Table 1. Initial LPDA Design

\begin{tabular}{|c|c|c|c|c|c|}
\hline Element \# & $\mathrm{L}_{\mathrm{n}}(\mathrm{m})$ & $\mathrm{h}_{\mathrm{n}}(\mathrm{m})$ & $\mathrm{X}_{\mathrm{n}}(\mathrm{m})$ & $\mathrm{D}_{\mathrm{n}}(\mathrm{m})$ & $\mathrm{d}_{\mathrm{n}}(\mathrm{m})$ \\
\hline 1 & 0.375 & 0.1875 & 0.0437 & 0.12 & 0.003 \\
\hline 2 & 0.3375 & 0.1688 & 0.0394 & 0.108 & 0.0027 \\
\hline 3 & 0.3038 & 0.1519 & 0.0354 & 0.0972 & 0.0024 \\
\hline 4 & 0.2734 & 0.1367 & 0.0319 & 0.0875 & 0.0022 \\
\hline 5 & 0.246 & 0.123 & 0.0287 & 0.0787 & 0.002 \\
\hline 6 & 0.2214 & 0.1107 & 0.0258 & 0.0709 & 0.0018 \\
\hline 7 & 0.1993 & 0.0996 & 0.0232 & 0.0638 & 0.0016 \\
\hline 8 & 0.1794 & 0.0897 & 0.0209 & 0.0574 & 0.0014 \\
\hline 9 & 0.1614 & 0.0807 & 0.0188 & 0.0517 & 0.0013 \\
\hline 10 & 0.1453 & 0.0726 & 0.0169 & 0.0465 & 0.0012 \\
\hline 11 & 0.1308 & 0.0654 & 0.0153 & 0.0418 & 0.001 \\
\hline 12 & 0.1177 & 0.0588 & 0.0137 & 0.0377 & 0.0009 \\
\hline 13 & 0.1059 & 0.053 & 0.0124 & - & 0.0008 \\
\hline
\end{tabular}

\section{Particle Swarm Optimization}

Kennedy and Eberhart[5] was the pioneer of Particle Swarm Optimization (PSO), who proposed this evolutionary algorithm which based on group intelligence. It is our common observation in our daily life that different species like birds, bees, etc. travel from one place to another for the search of food. Every individual particle, in our case is bird, has its own local best or pbest which is determined by its location in the swarm. On the other hand, each bird has some way of knowing the more concentration of food discover by the whole swarm. This location is known as global best or gbest. The gbest is the only location in the whole swarm where every bird desires to achieve its best position. For the optimum solution, there must be a fitness function which search in the entire solution space of swarm and produce single number of position[19].

For an optimum solution, random positions and velocities of the swarm particles are initialized to optimize the parameters. However, the restriction on the positions and the velocities of the particles is applied to keep the particles in search area to avoid the unrealistic solution. The optimized parameters of any problem define the dimensions of the problem. The updated positions and velocities are determined by the following equations.

$$
\begin{gathered}
X_{m+1}^{i}=X_{m}^{i}+V_{m+1}^{i} \\
V_{m+1}^{i}=V_{m}^{i}+c_{1} \times r_{1}\left(P_{m}^{i}-X_{m}^{i}\right)+c_{2} \times r_{2}\left(P_{m}^{g}-X_{m}^{i}\right)
\end{gathered}
$$

Where, $X_{m}^{i}$ is the particle position, $V_{m}^{i}$ is the particle velocity, $P_{m}^{i}$ is the best individual particle position, $P_{m}^{g}$ is the best swarm particle position and $m$ represents number of iterations in the whole swarm. $c_{1}$ and $c_{2}$ determines relative push of pbest and gbest in the entire swarm. $r_{1}$ and $r_{2}$ exists in the range of $(0,1)$. The velocities are scaled by inertia weight $w$ which consequently changes the direction of pbest and gbest for specific dimension.

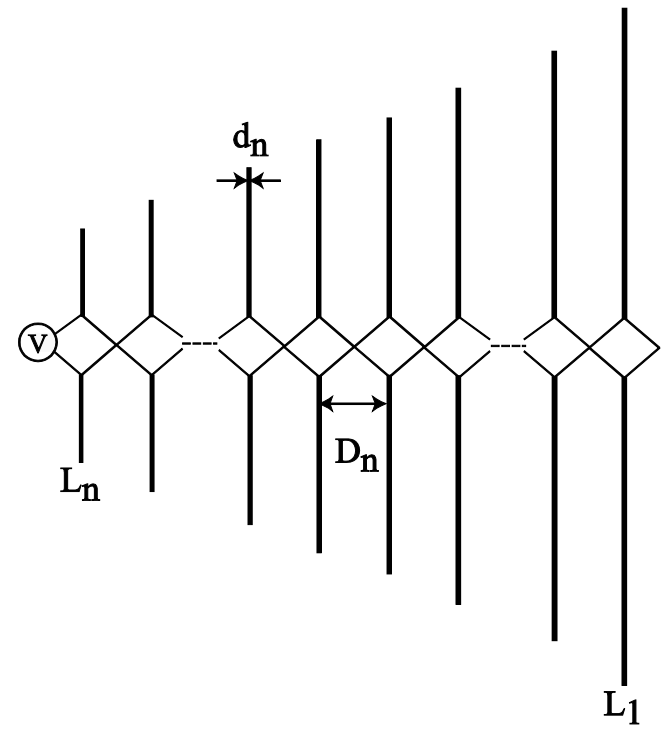

Figure 1. Geometry of Log Periodic Dipole Array

\section{Implementation of PSO}

PSO is implemented on 13 dipole elements of LPDA with the goal to reduce the computational cost of optimization algorithm to a considerable time. So, number of dimensions of the optimization is set as low as possible. The number of parameters to be optimized for a given problem defines dimensions of the entire swarm. The dimensions of the swarm are chosen to form only 13 design elements. In swarm, every particle is responsible to define the length of first element and rest of the lengths, spacing and diameters. Hence, the physical dimensions of optimized antenna are depending on the swarm particles which define the length of the first dipole element. The fitness function consists on a condition to improve gain as compared to the initial one with 
optimum VSWR. The minimum and maximum velocities are 0 and 0.2 , respectively, and the particles are in the range of[0.6-1.1]. The position and the velocity of the particle are updated by using eqs. (5) and (6). After simulation, we get an optimum design of LPDA which is shown in Table 2.

The optimum solution of gain and VSWR against these parameters is shown in Fig. 2-6. The comparison between the initial and optimized gain for reported frequency bands is shown in Fig. 2-5. It is clear from the figures that the presented optimization technique gives better response for gain than the initial one. From Fig. 6, it is observed that the initial mean VSWR reduce to its ideal value which is $\leq 1.5$.

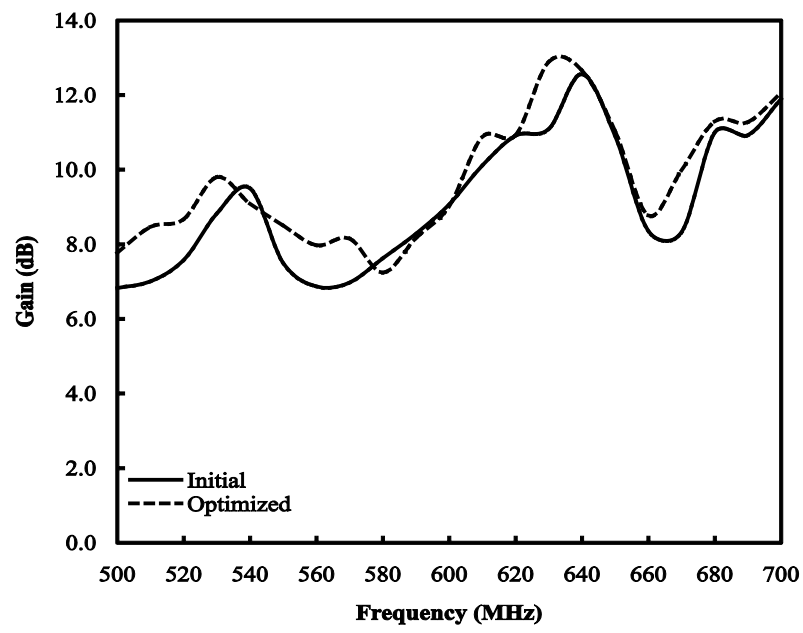

Figure 2. Initial and optimized gain for WiMAX frequency band

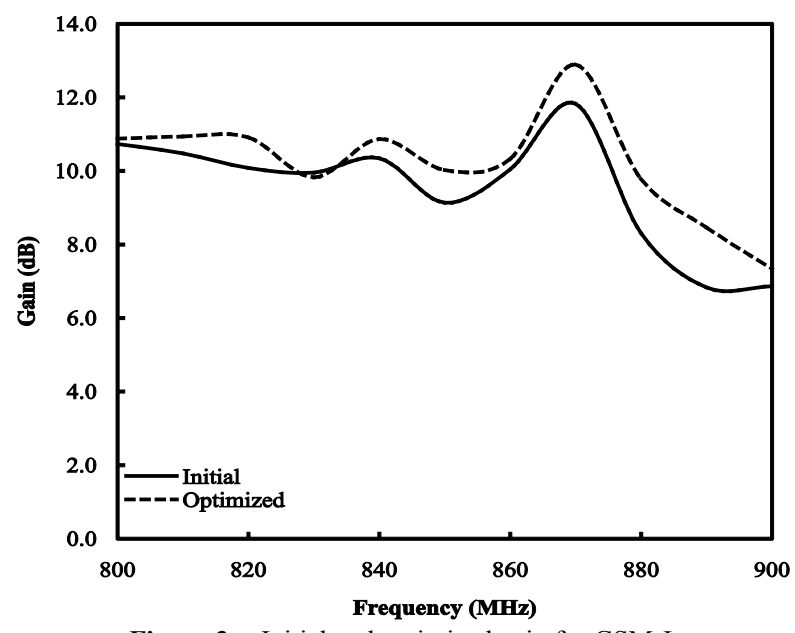

Figure 3. Initial and optimized gain for GSM-I

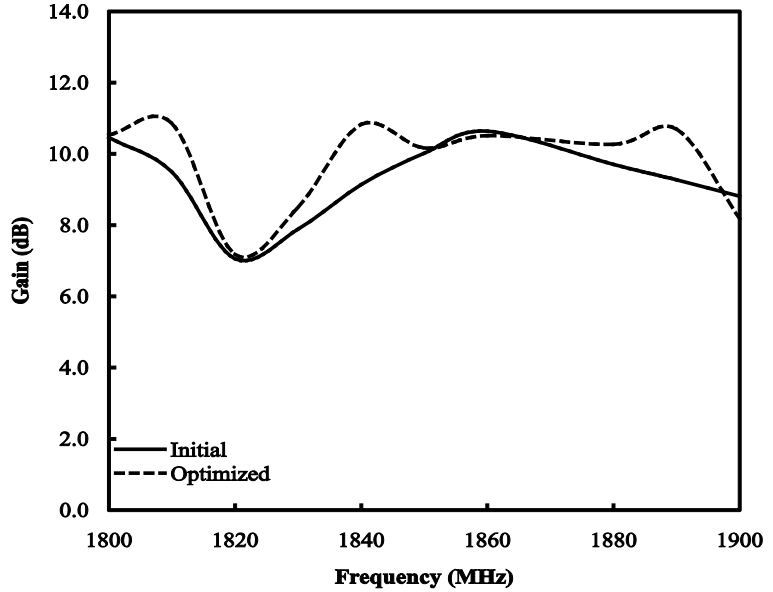

Figure 4. Initial and optimized gain for GSM-

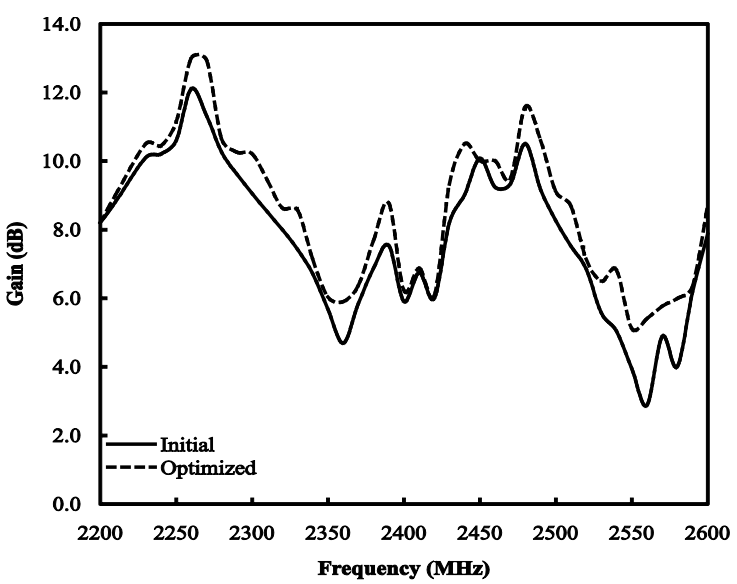

Figure 5. Initial and optimized gain for Bluetooth ${ }^{\circledR}$, Wi-Fi and $3 \mathrm{G}$ mobile communication bands

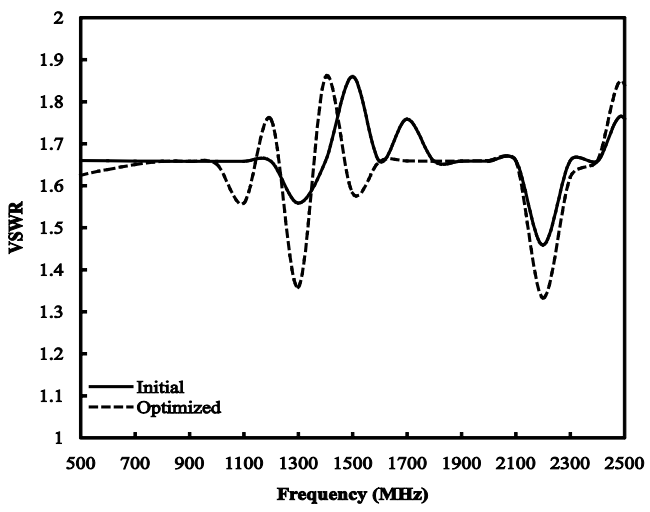

Figure 6. Initial and optimized VSWR

Table 2. Optimized LPDA Design Using PSO

\begin{tabular}{|c|c|c|c|c|c|}
\hline Element \# & $\mathrm{L}_{\mathrm{n}}(\mathrm{m})$ & $\mathrm{h}_{\mathrm{n}}(\mathrm{m})$ & $\mathrm{X}_{\mathrm{n}}(\mathrm{m})$ & $\mathrm{D}_{\mathrm{n}}(\mathrm{m})$ & $\mathrm{d}_{\mathrm{n}}(\mathrm{m})$ \\
\hline 1 & 0.2213 & 0.1106 & 0.0258 & 0.0708 & 0.0018 \\
\hline 2 & 0.1991 & 0.0996 & 0.0232 & 0.0637 & 0.0016 \\
\hline 3 & 0.1792 & 0.0896 & 0.0209 & 0.0573 & 0.0014 \\
\hline 4 & 0.1613 & 0.0806 & 0.0188 & 0.0516 & 0.0013 \\
\hline 5 & 0.1452 & 0.0726 & 0.0169 & 0.0465 & 0.0012 \\
\hline 6 & 0.1306 & 0.0653 & 0.0152 & 0.0378 & 0.001 \\
\hline 7 & 0.1176 & 0.0588 & 0.0376 & 0.0009 \\
\hline 8 & 0.1058 & 0.0529 & 0.0123 & 0.0305 & 0.0008 \\
\hline 10 & 0.0952 & 0.0476 & 0.0111 & 0.0274 & 0.0007 \\
\hline 11 & 0.0857 & 0.0429 & 0.01 & 0.0247 & 0.0006 \\
\hline 12 & 0.0771 & 0.0386 & 0.009 & 0.0222 & 0.0006 \\
\hline 13 & 0.0694 & 0.0347 & 0.0381 & - & 0.0005 \\
\hline
\end{tabular}




\section{Conclusions}

The LPDA is designed and then it is optimized by using stochastic technique called Particle Swarm Optimization (PSO). All work is done by writing a MATLAB routine and the results are shown. It is observed that the optimum solution for gain for WiMAX, GSM phase (I and II), Bluetooth ${ }^{\circledR}$ and $3 \mathrm{G}$ mobile communication bands has been improved up to $0.6 \mathrm{~dB}, 0.7 \mathrm{~dB}, 0.6 \mathrm{~dB}$ and $0.8 \mathrm{~dB}$, respectively. It is also observed that the unequal length, spacing and diameter of each dipole element is reduced as compared to the standard design. The VSWR is also reduced to its acceptable value which is $\leq 1.5$.

\section{REFERENCES}

[1] Isbell, D., "Log periodic dipole arrays", IRE Transactions on Antennas and Propagation, vol. 8, pp. 260-267, 1960.

[2] Carrel, R., "The design of log-periodic dipole antennas", IRE International Convention Record, vol. 9, pp. 61-75, 1961.

[3] Rumsey, V. H., "Frequency independent antennas", IRE International Convention Record, pp. 114-118, 1957.

[4] King, H., "Mutual impedance of unequal length antennas in echelon", IRE Transactions on Antennas and Propagation, vol. 5, pp. 303-313, 1957.

[5] Eberhart, R. and Kennedy, J., “A new optimizer using particle swarm theory", Proceedings of the $6^{\text {th }}$ International Symposium on Microwave Machine and Human Science, pp. 39-43, 1995.

[6] Pantoja, M. F., Bretones, A. R., Ruiz, F. G., Garcia, S. G. and Martin R. G., "Particle swarm optimization in antenna design optimization of log-periodic dipole arrays", IEEE Antennas and Propagation Magazine, vol. 49, pp. 34-47, 2007.

[7] Ronghong, J., Zhihao, Y., Geng, G. J., Fan, Y. and Jiajing, L., "Pattern synthesis of antennas based on a modified particle swarm optimization algorithm", Frontiers of Electrical and Electronic Engineering, vol. 2, pp. 454-458, 2007.

[8] Bayraktar, Z., Werner, P. L. and Werner, D. H., "The design of miniature three-element yadi uda arrays in particle swarm optimization", IEEE Antennas and Wireless Propagation Latters, vol. 5, pp. 22-26, 2006.

[9] Baskar, S., Alphones, A., Suganthan, P. N. and Liang, J. J., "Design of yagi antenna using comprehensive learning particle swarm optimization", IEEE Proceedings of Microwave, Antennas and Propagation, vol. 152, no. 5, pp. 340-346, 2005.

[10] Azaro, A. Natale, F. de, Donelli, M., Zeni, E. and Massa, A., "Synthesis of a prefractal dual-band monopole antenna for GPS applications", IEEE Antennas and Wireless Propagation Letters, vol. 5, pp.361-364, 2006.

[11] Jakensai, P. J., "Particle swarm optimization of reduced-size HF dipoles", 2006 IEEE International Symposium on Antennas and Propagation Digest, pp. 3547-3550, 2006.

[12] Liu, W., "Design of a multiband CPW-fed monopole antenna using a particle swarm optimization approach", IEEE Transacions on Antennas and Propagation, Ap-53, no. 10, pp. 327-329, 1997.

[13] Kim, Y. and Ling, H., "Equivalent circuit modeling of broadband antennas using vector fitting and particle swarm optimization", 2006 IEEE International Symposium on Antennas and Propagation Digest, pp. 3555-3558, 2006.

[14] Xu, S. and Samii, Y. R., "Multi-objective particle swarm optimization for high performance array and reflector antennas", 2006 IEEE Symposium on Antennas and Propagation Digest, pp. 3523-3296, 2006.

[15] Gies, D. and Samii, Y. R., "Particle swarm optimization (PSO) for reflector antenna shapping”, 2004 IEEE International Symposium on Antennas and Propagation Digest, pp. 2289-2292, 2004.

[16] Vito, G. D. and Stracca, G., "Comments on the design of log-periodic dipole antennas", IEEE Transactions on Antennas and Propagation, vol. 21, pp. 303-308, 1973.

[17] Bantin, C. and Balmain, K., "Study of compressed log-periodic dipole antennas", IEEE Transactions on Antennas and Propagation, vol. 18, pp. 195-203, 1970.

[18] Balanis, C. A., Antenna Theory and Design, $3^{\text {rd }}$ Edition.

[19] Robinson, J. and Samii, Y. R., "Particle swarm optimization in electromagnetic", IEEE Transactions on Antennas and Propagation, vol. 52, pp. 397-407, 2004. 